\title{
Genetic modification of cereals and increasing size of caryopsis
}

\author{
I. Erokhin \\ NBK LLC, Moscow, Russia \\ e-mail: i.erokhin@inbox.ru
}

Key words: caryopsis, cereals, ontogenesis, junk DNA, Genome Tree Theory

In accordance with the Genome Tree Theory [1], the genome of a multicellular organism is structured (in terms of graphs theory) in the form of an oriented binary tree, whose tops are represented by similar logical elements "genome tree cycle step", and arcs are the transfers of control between the "steps". Each cell of the multicellular organism has its step in its Genome Tree. It is controlled by the step of the mother cell, initiates performance of the "cell program" which determines its development and division, and returns control upon its completion and transfers management to the steps of daughter cells. The cell program is a network (multi-level embedded Dijkstra loop), where regulatory genes, as well as structural ones, are used in a certain order. The step of the Cell Program may be represented by a couple of non-coding regulatory genes with identical complex promotors, and the Genome Tree step - by three non-coding regulatory genes. Separate steps of the Genome Tree may also be represented by protein-coding regulatory genes.

Transcription of the non-coding regulatory gene is supposedly initiated with the help of the nucleoprotein formed with general transcription factors of Pol III polymerase that contains a short RNA transcribed from another non-coding regulatory gene. One of the sites (ligand) of this short RNA should be complimentary to some site (acceptor) of promotor of the initiated regulatory gene. Another site (emitter) of the initiated regulatory gene encodes the new ligand in the transcribed short RNA. This new ligand may help to initiate another regulatory non-coding gene. Thus, control is transmitted between regulatory genes of Cell Programs and Genome Tree.

The size and shape of caryopsis of cereals are determined by the relevant branch of the Genome Tree, dominant allele of triploid genome of endosperm cells. In particular, its size (number of cells that form an endosperm) depends on the number of steps that form this branch of the Genome Tree. Adding another step to this branch leads to an increase in the number of cells and, consequently, the size of caryopsis. The number of added cells depends on the position of the additional step in the Genome Tree branch. The closer is the added step to the root (in terms of graphs theory) of the branch responsible for endosperm formation, the more new cells are added. Adding a step in extreme tops of the Genome Tree leads to an insignificant increase in the number of new cells. The endosperm properties depend on the activated Cell Programs. To insert an additional step between the two available (previous and subsequent) steps requires adding to the genome three extra non-coding regulatory genes with identical complex promotors (or modifying the three inactive genes that exist). An additional step in other branches of the Genome Tree will lead to increase in sizes of the relevant organs or the whole plant. Such accidental mutations of regulatory genes are responsible for a variety of sizes and shapes of Angiospermae and Gramíneae, among others.

\section{References}

1. Erokhin I. (2016) Genome tree theory. International Conference on the Mathematical Modeling and High-Performance Computing in Biomedicine and Biotechnology, August 29-September 2, Novosibirsk, Russia. 